\title{
Nebivolol Prevents the Increase of Asymmetric Dimethylarginine and Oxidants in Hyperhomocysteinemic Rats
}

\author{
Mustafa Ahmet Huyut \\ Department of Cardiology, Faculty of Medicine, Bezmialem Vakif University, Istanbul, Turkey \\ ORCID: \\ Mustafa Ahmet Huyut: https://orcid.org/0000-0001-8740-1429
}

\section{Abstract}

Objective: The objective of this study was to determine nebivolol's inhibitory effect on endothelial dysfunction in hyperhomocysteinemic rats, based on heart pathology and biochemical analysis of serum samples. Methods: Male Wistar albino rats weighing between $200 \mathrm{~g}$ and $450 \mathrm{~g}$ were randomly divided into four groups of equal number $(n=7)$ as follows: control group, nebivolol group, methionine group, and methionine + nebivolol group. After 28 days, homocysteinemia (Hcy), asymmetric dimethylarginine (ADMA), malondialdehyde (MDA), glutathione (GSH), glutathione peroxidase (GPx), glutathione reductase (GR), superoxide dismutase (SOD), and catalase (CAT) levels were measured in blood samples and compared between groups. Each rat's hearts were dissected to observe cardiomyocyte degeneration; findings were compared between groups. Results: Moderate hyperHcy (hHcy) (Hcy $35.62 \pm 7.60 \mu \mathrm{mol} / \mathrm{L})$ was noted in methionine group $(P<0.001)$. The levels of the antioxidant molecules CAT, GSH, GPx, GR, and SOD were lower, and the levels of the oxidant molecules ADMA, Hcy, and MDA were higher in methionine group $(P<0.001)$. A decrease in antioxidants and also increase in oxidants did not occur in the methionine + nebivolol group $(P<0.001)$. Cardiomyocyte degeneration was more severe in methionine group $(P=0.01)$. Conclusion: Endothelial dysfunction induced through short-term hHcy can be prevented through the administration of nebivolol. Nebivolol can prevent elevation of the Hcy levels, and hHcy might cause cardiomyocyte degeneration.

Keywords: Antioxidants, asymmetric dimethylarginin, hyperhomocysteinemia, nebivolol, oxidants

\section{INTRODUCTION}

Hyperhomocysteinemia (hHcy) is a serious cardiovascular risk factor that occurs due to pharmacological or genetic causes. ${ }^{[1,2]}$ Impaired endothelial function induced by hHcy is a significant cause of morbidity and mortality. Many studies have investigated how to prevent impairment of endothelial function induced by hHcy using vitamins and pharmaceutical agents.

The association between hHcy and cardiovascular diseases was first described by McCully in 1969. McCully observed widespread arterial thrombosis and atherosclerosis. ${ }^{[3,4]}$ A prospective study by Page et al. ${ }^{[5]}$ that carried out among women reported that hHcy was an independent risk factor for myocardial infarction.

Healthy endothelial cells detoxify Hcy through the production of nitric oxide (NO). During Hcy metabolism, Hcy combines with

Submission: 31-Aug-18 Accepted: 12-Oct-18

\begin{tabular}{|l|l|}
\hline \multicolumn{2}{|c|}{ Access this article online } \\
\hline Quick Response Code: & Website: \\
\hline & www.ijcva.com \\
\hline & \\
\hline
\end{tabular}

adenosine to form S-adenosylhomocysteine while decreasing the adenosine concentration. The decrease in adenosine plasma levels will result in vascular damage. ${ }^{[4,6]}$ Asymmetric dimethylarginine (ADMA) is the endogen inhibitor of the eNOs. Hcy is the most prominent factor that increases the level of ADMA. ${ }^{[7,8]}$ Long-term exposure to ADMA produces hypertensive damage in target organs and accelerates atherogenesis. ${ }^{\left[{ }^{9]}\right.}$ High ADMA level increased the risk of acute coronary events by 3.9 -fold. ${ }^{[10]}$

Nebivolol is a third-generation, highly selective $\beta 1$ blocker that promotes vasodilatation through increasing $\mathrm{NO}$ in the L-arginine-NO pathway. ${ }^{[11,12]}$ Moreover, unlike other

Address for correspondence: Dr. Mustafa Ahmet Huyut, Department of Cardiology, Faculty of Medicine, Gaziosmanpasa Hospital, Yeni Yuzyil University, Cukurcesme Caddesi No: 51 Istanbul, Turkey. E-mail:ahuyut@yahoo.com

This is an open access journal, and articles are distributed under the terms of the Creative Commons Attribution-NonCommercial-ShareAlike 4.0 License, which allows others to remix, tweak, and build upon the work non-commercially, as long as appropriate credit is given and the new creations are licensed under the identical terms.

For reprints contact: reprints@medknow.com

How to cite this article: Huyut MA. Nebivolol prevents the increase of asymmetric dimethylarginine and oxidants in hyperhomocysteinemic rats. Int J Cardiovasc Acad 2019;5:8-14. 
beta-blockers, nebivolol increases the NO level by activating eNOs, as it has an agonistic effect on $\beta 3$ receptors. ${ }^{[13]}$ To prevent hHcy and its harmful effects, Vitamin B12, Vitamin C, Vitamin $\mathrm{E}$, and folic acid have been used in trials with some success. ${ }^{[14-18]}$ The present study was, therefore, conducted to investigate the use of nebivolol for the prevention of hHcy and its harmful effects.

\section{Methods}

The study included 28 adult male Wistar albino rats weighed between 250 and $450 \mathrm{~g}$ that were obtained from the local University Experimental Animals Laboratory. The rats were randomly divided into four groups of equal number $(n=7)$, and housed in wired cages for 1 month under 12-h circadian rhythm conditions at $22^{\circ} \mathrm{C}-24^{\circ} \mathrm{C}$. All the groups were fed standard rat silage and urban drinking water ad libitum. The study protocol was approved by the local University Laboratory Animals Ethics Committee. This study was supported by the local University Scientific Research Projects Unit.

\section{Experimental model}

1. The control group $(n=7)$ : no medication was administered for 4 weeks. The rats received $1 \mathrm{~mL}$ of drinking water od through orogastric gavage to experience the stress of the gavage method.

2. Nebivolol group $(n=7)$ : the rats received nebivolol $10 \mathrm{mg} / \mathrm{kg} / \mathrm{day}$ od through orogastric gavage for 4 weeks

3. Methionine group $(n=7)$ : the rats received methionine (M9625 Sigma-Aldrich L-Methionine reagent grade, $\geq 98 \%$ [thin layer chromatography [TLC]) $1 \mathrm{mg} / \mathrm{kg} /$ day od through orogastric gavage for 4 weeks

4. Methionine + nebivolol $(n=7)$ : the rates received methionine $1 \mathrm{~g} / \mathrm{kg} /$ day + nebivolol $10 \mathrm{mg} / \mathrm{kg} /$ day od through orogastric gavage for 4 weeks. Nebivolol was administered $2 \mathrm{~h}$ after the administration of methionine.

Body weight was measured at baseline, and then every week for the adjustment of drug dosage. To achieve hHcy, L-methionine (M9625 Sigma-Aldrich L-Methionine reagent grade, $\geq 98 \%[\mathrm{TLC}]$ ) was dissolved in phosphate-buffered

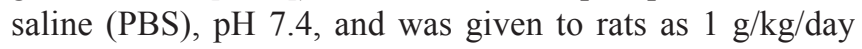
through oral gavage for 4 weeks. Nebivolol was obtained by crushing $5 \mathrm{mg}$ nexivol (Abdi İbrahim) tablets and dissolving in $10 \%$ dimethyl sulfoxide and was given as $10 \mathrm{mg} / \mathrm{kg} /$ day through oral gavage for 4 weeks. After 4 weeks, the rats were sacrificed under ether anesthesia, and blood samples were drawn into hemogram tubes with EDTA and standard biochemical tubes. Subsequently, hearts were dissected and immersed in $10 \%$ formaldehyde solution for histochemical examination. Blood samples were centrifuged at $4000 \mathrm{rpm}$ at $+4^{\circ} \mathrm{C}$ for $10 \mathrm{~min}$ and then stored at $-80^{\circ} \mathrm{C}$. Serum was used for the analysis of ADMA, Hcy, malondialdehyde (MDA), NO, catalase (CAT), superoxide dismutase (SOD), and glutathione (GSH), whereas plasma was used for the analysis of glutathione peroxidase (GPx) and glutathione reductase (GR).

\section{Biochemical examination}

ADMA (ng/mL) (USCN Life Science Inc., catalog no: $\mathrm{E} 91301 \mathrm{Ge})$ and Hcy $(\mu \mathrm{mol} / \mathrm{L})(\mathrm{Cusabio}$, catalog no. CSB-E13376r) levels were detected through the ELISA method using commercial kits. CAT $(\mathrm{U} / \mathrm{g})$ was measured through the ELISA method using a CAT assay kit (Cayman, catalog no. 7070002, USA). GR activity (U/g) was measured through ELISA using a GR assay kit (Cayman, catalog no. 703202, USA). GPx (U/g) was measured through the ELISA method using a GPx assay kit (Cayman, catalog no. 703102, USA). GSH was measured through the ELISA method using a GSH assay kit (Cayman, catalog no. 703002, USA). SOD was measured according to Sun et al. ${ }^{[19]}$ The method is based on the reduction of nitroblue tetrazolium through xanthine-xanthine oxidase, which is the producer of superoxide. SOD activity is expressed as $\mathrm{U} / \mathrm{mg}$ of serum protein. The level of serum protein was determined using the biuret method and an Abbott Architect C 8000 Autoanalyzer (Abbott, Abbott Park, IL, USA). MDA was measured using the Esterbauer method and a lipid peroxidation measurement method. ${ }^{[20]} \mathrm{MDA}$, which reacts with thiobarbituric acid at $90^{\circ} \mathrm{C}-95^{\circ} \mathrm{C}$, produces a pink chromogen. After $15 \mathrm{~min}$, the absorbance of the samples that were chilled quickly was read at $532 \mathrm{~nm}$, and the results expressed as $\mathrm{nmol} / \mathrm{mL}$.

\section{Pathological examination}

Paraffin blocks were prepared from heart tissue samples. Sections $4-\mu \mathrm{m}$ thick were cut using a microtome. The sections were deparaffinized and collected on glass slides. After staining with $\mathrm{H}$ and $\mathrm{E}$ (Gul Biology Laboratory, dye for connective and supportive tissue of animals, catalog no. 5089, Istanbul, Turkey), the slides were examined under a light microscope. All samples were evaluated by blinded pathologist. For heart tissue evaluation, myocyte damage was semiquantitatively scored with respect to necrosis, myocyte cell swelling, and detection of fibrosis, according to the scale shown below.

The scoring system scale as follows:

$(-)$ : No change

$(+)$ : Slight change

$(++)$ : Moderate change

$(+++)$ : Severe change.

\section{Statistical analysis}

Statistical analysis was performed by using the Statistical Package for the Social Sciences (SPSS) version 14.0 for Windows (SPSS, Inc., Chicago. IL, USA). The groups were compared inter se and with the control group. The Kolmogorov-Smirnov test was used to assess the compliance of the quantitative values to a normal distribution. Single-direction variance analysis was used to compare normally distributed quantitative data between groups, and Tukey's honestly significant difference or Dunnett's T3 test was used for multiple comparisons between groups, according to the Levene homogeneity test's statistics. Descriptive statistics are shown as mean \pm standard deviation. The Kruskal-Wallis 
variance analysis was used to compare data not distributed normally between the groups. Descriptive statistics are shown as median $\left(25^{\text {th }}-75^{\text {th }}\right.$ percentile). The level of statistical significance was set at $P<0.05$.

\section{RESULTS}

\section{Biochemical findings}

Oxidants diagram was showed in Figure 1. The antioxidants diagram was shown in Figures 2 and 3. hHcy was more severe in the methionine group than in the other three groups $(P<0.001)$. The Hcy level was significantly lower in the methionine + nebivolol group than in the methionine group $(13.2 \pm 2.1$ vs. $35.6 \pm 7.6 \mu \mathrm{mol} / \mathrm{L})(P<0.001)$.

The plasma ADMA level was significantly higher in the methionine group than in the other groups $(P<0.001)$. In addition, the ADMA level was significantly lower in the methionine + nebivolol group than in the methionine group $(113.2 \pm 16.0$ vs. $225.7 \pm 42.2 \mathrm{ng} / \mathrm{mL})(P<0.001)$.

The plasma CAT level was significantly lower in the methionine group than in the other groups $(P<0.001)$ and was significantly higher in the methionine + nebivolol group than in the methionine group $(36.0 \pm 3.5$ vs. $23.7 \pm 6.1 \mathrm{U} / \mathrm{g})(P=0.006)$. In addition, the CAT level was higher in the nebivolol group than in the methionine + nebivolol group $(36.0 \pm 3.5$ vs. $49.0 \pm 5.7 \mathrm{U} / \mathrm{g})(P=0.003)$.

The GSH level was significantly lower in the methionine group than in the other groups $(P<0.001)$ and was significantly higher in the methionine + nebivolol group than in the methionine group $(1.0 \pm 0.1 \mathrm{vs}$ $0.4 \pm 0.1 \mu \mathrm{mol} / \mathrm{L})(P<0.001)$. In addition, the GSH level was lower in the methionine + nebivolol group than in the control group $(1.0 \pm 0.1$ vs. $1.4 \pm 0.3 \mu \mathrm{mol} / \mathrm{L})(P=0.03)$.

The plasma GPx level was significantly lower in the methionine group than in the other groups $(P<0.001)$ and was significantly higher in the methionine + nebivolol group than in the methionine group $(213.20 \pm 32.55$ vs. $122.54 \pm 11.56 \mathrm{U} / \mathrm{g})(P=0.001)$. In addition, the GPx level was lower in the methionine + nebivolol group than in the control group $(213.20 \pm 32.55$ vs. $424.13 \pm 38.43 \mathrm{U} / \mathrm{g})(P<0.001)$.

The plasma MDA level was significantly higher in the methionine group than in the other groups $(P<0.001)$ and was significantly lower in the methionine + nebivolol group than in the methionine group $(2.18 \pm 0.21 \mathrm{nmol} / \mathrm{mL}$ vs. $3.02 \pm 0.29 \mathrm{nmol} / \mathrm{mL})(P<0.001)$. In addition, the MDA level was higher in the methionine + nebivolol group than in the control group $(2.18 \pm 0.21 \mathrm{nmol} / \mathrm{mL}$ vs. $1.80 \pm 0.10 \mathrm{nmol} / \mathrm{mL})(P=0.015)$.

The plasma GR level was significantly lower in the methionine group than in the other groups $(P<0.001)$. In addition, the GR level was lower in the methionine + nebivolol group than in the control group but not significantly (184.56 vs. $273.86 \mathrm{U} / \mathrm{g})(P=0.081)$.

The plasma SOD level was significantly lower in the methionine group than in the other groups $(P=0.001)$. In addition, the SOD level was higher in the methionine + nebivolol group than in the control group but not significantly $(1.45 \mathrm{U} / \mathrm{mg}$ of

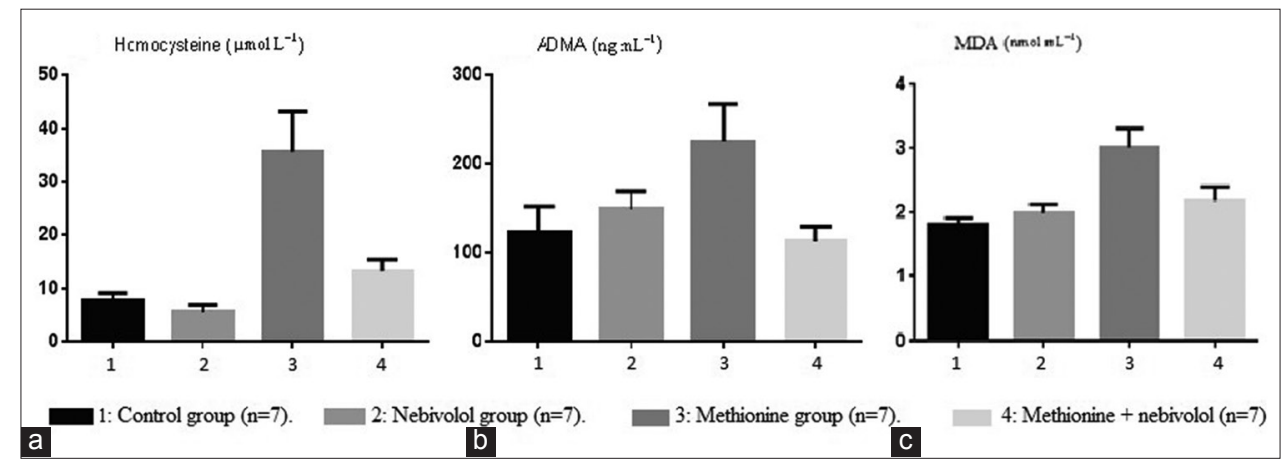

Figure 1: Homocysteinemia and oxidants diagram was showed. (a) Hyperhomocysteinemia, (b) plasma asymmetric dimethyl arginine, (c): and malondialdehyde levels were more severe in the methionine group than in the other groups $(P<0.001)$
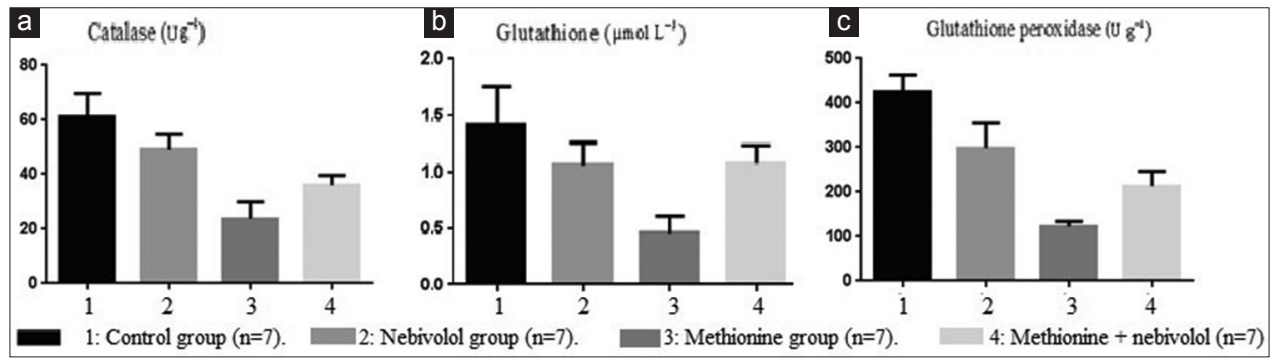

Figure 2: Antioxidants diagram was showed. (a) The plasma catalase, (b) glutathione, (c) and glutathione peroxidase levels were significantly lower in the methionine group than in the other groups $(P<0.001)$ 


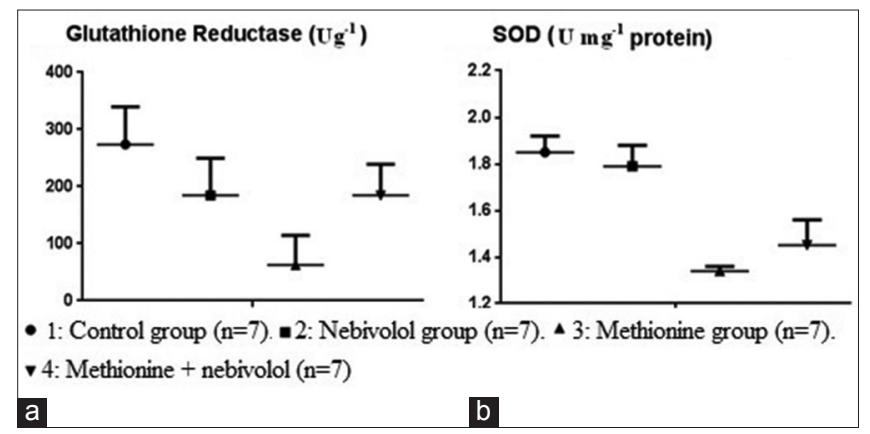

Figure 3: Antioxidants diagram which descriptive statistics for quantitative biochemical data not distributed normally (median given as $25^{\text {th }}-75^{\text {th }}$ percentile). (a) The plasma glutathione reductase level was significantly lower in the methionine group than in the other groups $(P<0.001)$. (b) The plasma superoxide dismutase level was significantly lower in the methionine group than in the other groups $(P=0.001)$

protein vs. $1.34 \mathrm{U} / \mathrm{mg}$ of protein) $(P=1)$. Moreover, the SOD level was lower in the methionine + nebivolol group than in the control group $(1.34 \mathrm{U} / \mathrm{mg}$ of protein vs. $1.85 \mathrm{U} / \mathrm{mg}$ of protein) $(P=0.007)$.

\section{Pathological finding}

Cardiomyocyte degeneration was significantly more severe in the methionine group than in the other groups $(P=0.01)$ [Figures 4 and 5]. Cardiomyocyte degeneration was less severe in the methionine + nebivolol group than in the control group but not significantly $(1.4 \pm 0.5$ vs. $1.8 \pm 1.0$ degeneration) $(P=1)$.

\section{DISCUSSION}

The present study showed that impaired endothelial function resulted from short-term hHcy could be prevented through the administration of nebivolol. In addition, the administration of nebivolol od $2 \mathrm{~h}$ after oral administration of methionine was observed to prevent an increase in the Hcy level. Different data such as tissue oxidant levels from these studies subgroups were published before. In our previous study, GSH, CAT, MDA, and SOD activities in the tissues were determined. The luminal cross-sectional area (LCSA), total cross-sectional area (TCSA), and intima-media thickness (IMT) were measured in the thoracic aorta. Nebivolol treatment decreased high MDA levels in the brain, heart, and liver tissues. The level of GSH was higher in the heart, brain, and kidney tissues of the nebivolol + hHcy group. The activity of CAT increased only in the kidney tissue of the nebivolol + hHcy group, and the activity of SOD was increased in all the tissues in this group. However, serum levels did not measure, and heart tissue did not investigate. Increased IMT and TCSA in the nebivolol + hHcy group were significantly decreased after nebivolol administration. The LCSA was higher in the hHcy group than the control group. ${ }^{[21]}$

In this study, according to the present biochemical findings, concomitant administration of nebivolol + methionine can prevent impaired endothelial function induced by short-term hHcy. In the present study, cardiomyocyte degeneration was more severe in the methionine group based on histopathological findings.
L-methionine was administered at $1 \mathrm{~g} / \mathrm{kg} / \mathrm{day}$, as reported by Cao et al. ${ }^{[22,23]}$ In the present study, nebivolol was administered at $10 \mathrm{mg} / \mathrm{kg} / \mathrm{day}$, as the efficiency and safety of this dose are well known. ${ }^{[24,25]}$ In the difference between groups, hHcy was statistically significant $(P<0.001)$. The Hcy level in methionine + nebivolol group significantly lower than in the methionine group $(P=0.001)$, which indicates that the administration of nebivolol $2 \mathrm{~h}$ after the administration of methionine can prevent an increase in the Hcy level.

Nebivolol increases NO through the activation of eNOs and its $\beta 3$-receptor agonistic effect, which is distinct from other beta-blockers. ${ }^{[13]}$ Nebivolol increases total antioxidant capacity. In the present study, the highest ADMA level was observed in the methionine group, which had a high Hcy level. The ADMA level was $123.8 \pm 28.5 \mathrm{ng} / \mathrm{mL}$ in the control group versus $225.7 \pm 42.2 \mathrm{ng} / \mathrm{mL}$ in the methionine group, and the difference was statistically significant $(P<0.001)$.

An elevated ADMA level is an indicator of impaired endothelial function. In addition, the ADMA level can be used for the prediction of cardiovascular morbidity and mortality. The impaired endothelial function is indicative of the early stage of atherosclerosis. ${ }^{[26]}$ Zoccali et al.$^{[27]}$ reported that a $2-\mathrm{mmol} / \mathrm{L}$ increase in the plasma ADMA level increases the risk of cardiovascular disease by $37 \%$. In a study by Valkonen et al., ${ }^{[10]}$ it was observed that an increase in the ADMA was associated with a 3.9-fold increase in the risk of acute coronary events. In earlier studies, Vitamin B12, Vitamin C, Vitamin E, and folic acid were used to prevent hHcy and these pathologies, and partial success has been achieved. ${ }^{[14-18]}$

A decrease in ADMA decreases oxidative stress and cellular damage; thus, protein breakdown is prevented and protein synthesis continues. When there is a need for cysteine Hcy is converted to cystathionine with cystathionine beta-synthase through the transsulfuration pathway and is subsequently hydrolyzed to cysteine and excreted in urine or used in protein synthesis. ${ }^{[2]}$ Hcy is metabolized through remethylation and transsulfuration. When remethylation reactions are saturated or there is a need for cysteine Hcy is hydrolyzed to cysteine through the transsulfuration pathway. ${ }^{[2]}$ Cysteine is a rate-limiting substrate; specifically, Hcy breakdowns 


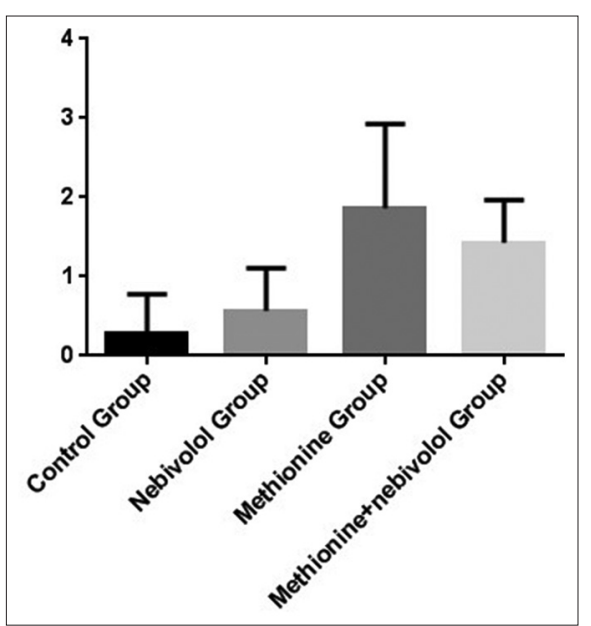

Figure 4: A significant difference in cardiomyocyte degeneration between the methionine group and control group was observed $(P=0.01)$

to form cysteine and an increase in the serum Hcy level is inhibited. Cysteine formed through transsulfuration of Hcy is used for GSH synthesis; therefore, nebivolol might inhibit an increase in Hcy through these physiologic pathways and others yet to be discerned. The metabolism of ADMA and Hcy shares common pathways. Hcy destabilizes the structure of proteins and increases proteolysis by increasing oxidative stress. ${ }^{[28]}$ On the other hand, the enzyme dimethylarginine dimethylaminohydrolase metabolizes ADMA. Hcy also inhibits this enzyme's activity and causes an increase in the ADMA concentration. ${ }^{[28]}$

Although the methionine + nebivolol group received methionine, the administration of nebivolol the same day might have inhibited an increase in the ADMA level, which might have been due to the lack of increase in the Hcy level. Nebivolol can prevent methionine-induced elevation of ADMA between the methionine group and the methionine + nebivolol group $(P<0.001)$. There was a positive correlation between oxidative stress and the CAT activity. Wyse et al. ${ }^{[18]}$ reported that Hcy administration reduced CAT activity in rats. CAT activity was significantly lower in the methionine group than in the methionine + nebivolol group $(P=0.006)$. Even though the methionine + nebivolol group received methionine, the concomitant administration of nebivolol prevented a decrease in the CAT activity.

Similar to our study, Dimitrova et al. ${ }^{[29]}$ reported that the myocardial GSH level was reduced significantly in rats that received Hcy. In the present study, the GSH level was significantly lower in the methionine group than in the other groups, which might indicate that oxidative stress was induced by hHcy in the methionine group. The significant difference in the GSH level was between the present study's methionine group and methionine + nebivolol group $(P<0.001)$.

A significant difference in GPx levels was observed between methionine group and control group $(P<0.001)$

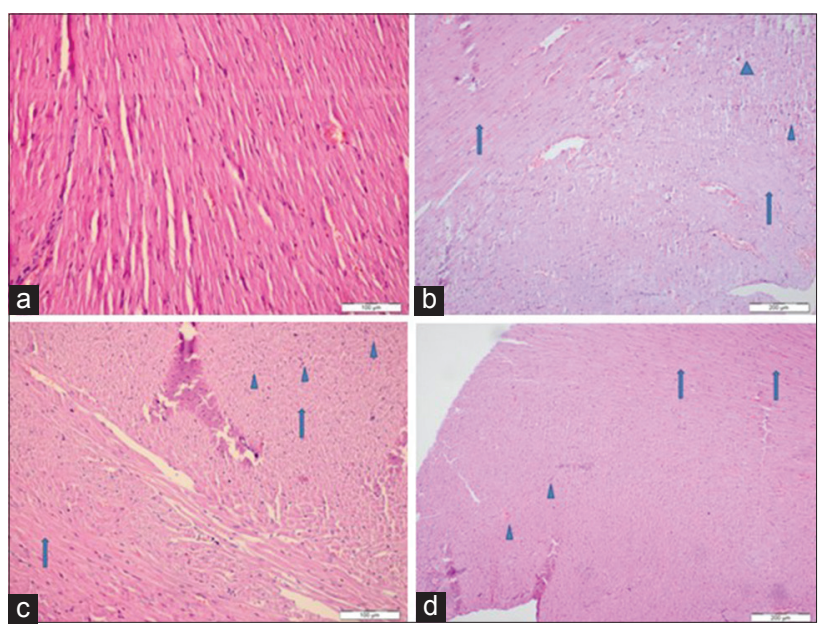

Figure 5: The arrow shows loss of nucleus. The triangle shows degeneration in muscle fiber (a) Control group heart tissue stained with $\mathrm{H}$ and $\mathrm{E}$ at $\times 200$ (normal). (b) Methionine group heart tissue stained with $\mathrm{H}$ and $\mathrm{E} \times 100$ (+3 degeneration). (c) Methionine group heart tissue stained with $\mathrm{H}$ and $\mathrm{E}$ at $\times 200(+3$ degeneration). (d) Methionine + nebivolol group heart tissue stained with $\mathrm{H}$ and $\mathrm{E}$ at 100 (+2 degeneration)

similar to Gilad's study. ${ }^{[30]}$ The significant difference in the GPx level between the methionine group and the methionine + nebivolol group $(P=0.001)$, which might have been associated with the difference in the Hcy level between two groups and the positive effects of nebivolol on total antioxidant capacity.

An elevated MDA level can be considered an indicator of cell damage and lipid peroxidation. ${ }^{[31,32]}$ Yüce and Aksakal ${ }^{[33]}$ examined the effect of Vitamin $\mathrm{E}$ in hyperhomocysteinemic rats and reported that the MDA level was higher in hyperhomocysteinemic rats. Similarly, in the present study, the MDA level was significantly higher in the methionine group than in the control group, which might have been an indicator of oxidative stress caused by an elevated Hcy level. Moreover, the MDA level was significantly higher in the methionine group than in the methionine + nebivolol group $(P<0.001)$. The present findings may show that the administration of nebivolol, the same day as the administration of methionine significantly inhibited an increase in the MDA level. Furthermore, in the present study, the GR level was significantly lower in the methionine group than in the control group $(P<0.001)$.

Yamamoto et al. ${ }^{[34]}$ observed that Hcy reduced the efficiency of SOD and damaged endothelial heparan sulfate proteoglycan and decrease the defensive ability of endothelial cells against free radicals. In the present study, the SOD level was significantly lower in the methionine group than in the control group $(P=0.001)$, which might have been due to elevated oxidative stress secondary to a high Hcy level.

Zivkovic et al. dissected rat hearts and perfused with Hcy in an organ bath, and showed that the left ventricle functions were destroyed. ${ }^{[35]}$ They suggested that the mechanism of action was the toxic effect of the increase in total oxidative capacity 
induced by impaired endothelium function and suppressed NO bioactivity that resulted from an elevated Hcy level. There was a significant difference in cardiomyocyte degeneration between the methionine group and control group $(P=0.01)$. hHcy might be caused cardiomyocyte cell degeneration. Chen et al.$^{[36]}$ observed a reduction in systolic function in rats with $\mathrm{hHcy}$, and an increased in the size of cardiomyocytes and an increase in intracellular fibrosis in rat heart pathological sections. Although maximum cardiomyocyte degeneration was noted in the methionine group, we could not show any significant protection with nebivolol administration.

The present study's limitations are having a small study population, and time was restricted for 4-week duration. Larger scale and longer durations are required to more clearly observe histopathological and biochemical changes.

\section{Study limitations}

To generalize these results and obtain significant histopathological findings, more clinical studies of longer duration and larger scale are necessary to generalize the present findings.

\section{Conclusion}

The present findings show that the impairment of endothelial function induced by short-term hHcy can be prevented by the administration of nebivolol. In addition, the administration of nebivolol $2 \mathrm{~h}$ after administration of methionine was observed to prevent an increase in the Hcy levels. The present study observes that impaired endothelial function due to short-term hHcy can be prevented by the concomitant administration of nebivolol.

\section{Acknowledgments}

The author would like to thank Adnan Menderes University Scientific Research Projects Unit No TPF-12010, Aydin, Turkey, for supporting this study and providing technical facilities for the work. Also author thanks for their valuable contributions to Cagdas Akgüllü, Murat Boyacioglu, Aslıhan Karul and Ibrahim Meteoglu.

\section{Financial support and sponsorship}

This study was funded by Adnan Menderes University Scientific Research Projects Unit No TPF-12010, Aydin, Turkey.

\section{Conflicts of interest}

There are no conflicts of interest.

\section{RefEREnCeS}

1. Obeid R. The metabolic burden of methyl donor deficiency with focus on the betaine homocysteine methyltransferase pathway. Nutrients 2013;5:3481-95.

2. Audelin MC, Genest J Jr. Homocysteine and cardiovascular disease in diabetes mellitus. Atherosclerosis 2001;159:497-511.

3. Cook JW, Taylor LM, Orloff SL, Landry GJ, Moneta GL, Porter JM. Homocysteine and arterial disease. Experimental mechanisms. Vascul Pharmacol 2002;38:293-300.

4. Joseph J, Loscalzo J. Methoxistasis: Integrating the roles of homocysteine and folic acid in cardiovascular pathobiology. Nutrients 2013;5:3235-56

5. Page JH, Ma J, Chiuve SE, Stampfer MJ, Selhub J, Manson JE, et al. Plasma total cysteine and total homocysteine and risk of myocardial infarction in women: A prospective study. Am Heart J 2010;159:599-604.

6. Memişoğulları R, Akçay F. Biochemical mechanisms in hyperhomocysteinemia. Türk Klin Biyokim Derg 2004;2:41-9.

7. Böger RH. Asymmetric dimethylarginine (ADMA): A novel risk marker in cardiovascular medicine and beyond. Ann Med 2006;38:126-36.

8. Bouras G, Deftereos S, Tousoulis D, Giannopoulos G, Chatzis G, Tsounis D, et al. Asymmetric dimethylarginine (ADMA): A promising biomarker for cardiovascular disease? Curr Top Med Chem 2013;13:180-200.

9. Kielstein JT, Impraim B, Simmel S, Bode-Böger SM, Tsikas D, Frölich JC, et al. Cardiovascular effects of systemic nitric oxide synthase inhibition with asymmetrical dimethylarginine in humans. Circulation 2004;109:172-7.

10. Valkonen VP, Päivä H, Salonen JT, Lakka TA, Lehtimäki T, Laakso J, et al. Risk of acute coronary events and serum concentration of asymmetrical dimethylarginine. Lancet 2001;358:2127-8.

11. Mangrella M, Rossi F, Fici F, Rossi F. Pharmacology of nebivolol. Pharmacol Res 1998;38:419-31.

12. Ignarro LJ, Byrns RE, Trinh K, Sisodia M, Buga GM. Nebivolol: A selective beta (1)-adrenergic receptor antagonist that relaxes vascular smooth muscle by nitric oxide- and cyclic GMP-dependent mechanisms. Nitric Oxide 2002;7:75-82.

13. Khan MU, Zhao W, Zhao T, Al Darazi F, Ahokas RA, Sun Y, et al. Nebivolol: A multifaceted antioxidant and cardioprotectant in hypertensive heart disease. J Cardiovasc Pharmacol 2013;62:445-51.

14. Holst-Schumacher I, Monge-Rojas R, Cambronero-Gutiérrez P, Brenes G. Genetic, dietary, and other lifestyle determinants of serum homocysteine levels in young adults in Costa Rica. Rev Panam Salud Publica 2005;17:263-70.

15. Savage DG, Lindenbaum J, Stabler SP, Allen RH. Sensitivity of serum methylmalonic acid and total homocysteine determinations for diagnosing cobalamin and folate deficiencies. Am J Med 1994;96:239-46.

16. Fakhrzadeh H, Ghotbi S, Pourebrahim R, Nouri M, Heshmat R, Bandarian F, et al. Total plasma homocysteine, folate, and Vitamin B12 status in healthy Iranian adults: The Tehran homocysteine survey (2003-2004)/a cross-sectional population based study. BMC Public Health 2006;6:29.

17. Myles PS, Chan MT, Forbes A, Leslie K, Paech M, Peyton P, et al. Preoperative folate and homocysteine status in patients undergoing major surgery. Clin Nutr 2006;25:736-45.

18. Wyse AT, Zugno AI, Streck EL, Matté C, Calcagnotto T, Wannmacher $\mathrm{CM}$, et al. Inhibition of $\mathrm{Na}(+), \mathrm{K}(+)$-ATPase activity in hippocampus of rats subjected to acute administration of homocysteine is prevented by Vitamins $\mathrm{E}$ and $\mathrm{C}$ treatment. Neurochem Res 2002;27:1685-9.

19. Sun Y, Oberley LW, Li Y. A simple method for clinical assay of superoxide dismutase. Clin Chem 1988;34:497-500.

20. Esterbauer H, Cheeseman KH. Determination of aldehydic lipid peroxidation products: Malonaldehyde and 4-hydroxynonenal. Methods Enzymol 1990;186:407-21.

21. Akgullu C, Huyut MA, Boyacioglu M, Guleş O, Eryilmaz U, Hekim T, et al. Nebivolol to attenuate the effects of hyper-homocysteinaemia in rats. Atherosclerosis 2015;240:33-9.

22. Cao L, Lou X, Zou Z, Mou N, Wu W, Huang X, et al. Folic acid attenuates hyperhomocysteinemia-induced glomerular damage in rats. Microvasc Res 2013;89:146-52

23. Xu Y, Tian Y, Wei HJ, Dong JF, Zhang JN. Methionine diet-induced hyperhomocysteinemia accelerates cerebral aneurysm formation in rats. Neurosci Lett 2011;494:139-44.

24. Cosentino F, Bonetti S, Rehorik R, Eto M, Werner-Felmayer G, Volpe M, et al. Nitric-oxide-mediated relaxations in salt-induced hypertension: Effect of chronic beta1-selective receptor blockade. J Hypertens 2002;20:421-8.

25. Sorrentino SA, Doerries C, Manes C, Speer T, Dessy C, Lobysheva I, et al. Nebivolol exerts beneficial effects on endothelial function, early 
endothelial progenitor cells, myocardial neovascularization, and left ventricular dysfunction early after myocardial infarction beyond conventional $\beta 1$-blockade. J Am Coll Cardiol 2011;57:601-11.

26. Satılmışoğlu H, Ozhan H, Albayrak S, Kaya A, Erden I, Yazıcı M. Relation of asymmetric dimethylarginine levels with conventional risk score systems in the healthy subjects with positive family history for coronary artery disease. Anadolu Kardiyol Derg 2011;11:114-8.

27. Zoccali C, Bode-Böger S, Mallamaci F, Benedetto F, Tripepi G, Malatino L, et al. Plasma concentration of asymmetrical dimethylarginine and mortality in patients with end-stage renal disease: A prospective study. Lancet 2001;358:2113-7.

28. Wanby P, Brattström L, Brudin L, Hultberg B, Teerlink T. Asymmetric dimethylarginine and total homocysteine in plasma after oral methionine loading. Scand J Clin Lab Invest 2003;63:347-53.

29. Dimitrova KR, DeGroot KW, Pacquing AM, Suyderhoud JP, Pirovic EA, Munro TJ, et al. Estradiol prevents homocysteine-induced endothelial injury in male rats. Cardiovasc Res 2002;53:589-96.

30. Gilad E, Cuzzocrea S, Zingarelli B, Salzman AL, Szabó C. Melatonin is a scavenger of peroxynitrite. Life Sci 1997;60:PL169-74.
31. Marnett LJ. Lipid peroxidation-DNA damage by malondialdehyde. Mutat Res 1999;424:83-95.

32. Buddi R, Lin B, Atilano SR, Zorapapel NC, Kenney MC, Brown DJ, et al. Evidence of oxidative stress in human corneal diseases. J Histochem Cytochem 2002;50:341-51.

33. Yüce A, Aksakal M. Effect of Melatonin on Homocysteine-Induced Changes in Oxidant-Antioxidant Systems and Coronary Arteries in Rats. FÜ Sağlık Bil Derg 2006;20:189-97.

34. Yamamoto M, Hara H, Adachi T. Effects of homocysteine on the binding of extracellular-superoxide dismutase to the endothelial cell surface. FEBS Lett 2000;486:159-62.

35. Zivkovic V, Jakovljevic V, Djordjevic D, Vuletic M, Barudzic N, Djuric D. The effects of homocysteine-related compounds on cardiac contractility, coronary flow, and oxidative stress markers in isolated rat heart. Mol Cell Biochem 2012;370:59-67.

36. Chen FY, Guo YH, Gao W, Feng XH, Chen L, Tang CS, et al. Effect of hyperhomocysteinemia on cardiac remodeling in rats. Beijing Da Xue Xue Bao Yi Xue Ban 2006;38:179-83. 